\title{
Checkerboard order in the $t-J$ model on the square lattice
}

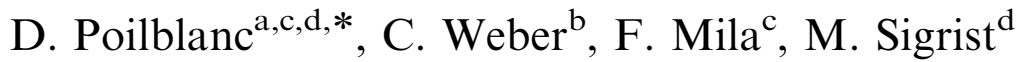 \\ ${ }^{a}$ Laboratoire de Physique Théorique UMR 5152, C.N.R.S. \& Université de Toulouse, F-31062 Toulouse, France \\ ${ }^{\mathrm{b}}$ Institut Romand de Recherche Numérique en Physique des Matériaux (IRRMA), PPH-Ecublens, CH-1015 Lausanne, Switzerland \\ ${ }^{\mathrm{c}}$ Institute of Theoretical Physics, Ecole Polytechnique Fédérale de Lausanne, BSP 720, CH-1015 Lausanne, Switzerland \\ ${ }^{\mathrm{d}}$ Theoretische Physik, ETH-Hönggerberg, 8093 Zürich, Switzerland
}

Available online 30 October 2006

\begin{abstract}
We propose that the inhomogeneous patterns seen by STM in some underdoped superconducting cuprates could be related to a bondorder-wave instability of the staggered flux state, one of the most studied "normal" state proposed to compete with the d-wave RVB superconductor. A checkerboard pattern is obtained by a Gutzwiller renormalized mean-field theory of the $t-J$ model for doping around $\frac{1}{8}$. It is found that the charge modulation is always an order of magnitude smaller than the bond-order modulations. This is confirmed by an exact optimization of the wavefunction by a variational Monte Carlo scheme. The numerical estimates of the order parameters are, however, found to be strongly reduced w.r.t their mean-field values.
\end{abstract}

(C) 2006 Elsevier B.V. All rights reserved.

PACS: 74.72.- $\mathrm{h} ;$ 71.10.Fd

Keywords: Cuprate superconductors; Pseudogap phase; $t-J$ model

\section{Introduction}

With constantly improving resolution of experimental techniques, novel features in the global phase diagram of high- $T_{\mathrm{c}}$ cuprate superconductors have emerged. One of the most striking is the observation by scanning tunnelling microscopy (STM), in the pseudogap phase of underdoped $\mathrm{Bi}_{2} \mathrm{Sr}_{2} \mathrm{CaCu}_{2} \mathrm{O}_{8+\delta}$ [1] and $\mathrm{Ca}_{2-x} \mathrm{Na}_{x} \mathrm{CuO}_{2} \mathrm{Cl}_{2}$ single crystals [2], of a form of local electronic ordering, with a spatial period close to four lattice spacings. These observations raise important theoretical questions about the relevance of such structures in the framework of strongly correlated models. Here, we analyze the stability of new inhomogeneous phases [3-5] which can compete in certain conditions with the d-wave superconducting RVB state [6]. Both mean-field [3] (MF) and numerical variational Monte Carlo results [5] (VMC) will be summarized here.

*Corresponding author. Laboratoire de Physique Théorique UMR 5152, C.N.R.S. \& Université de Toulouse, F-31062 Toulouse, France. Tel.: + 561556039 ; fax: +561556065 .

E-mail address: didier.poilblanc@irsamc.ups-tlse.fr (D. Poilblanc).

$U R L:$ http://www.lpt.ups-tlse.fr/.

\section{Renormalized MF theory}

We describe the doped antiferromagnet by a $t-J$ model, $H=-t \sum_{\langle i j\rangle \sigma}\left(c_{i, \sigma}^{\dagger} c_{j, \sigma}+\right.$ h.c. $)+J \sum_{\langle i j\rangle} \mathbf{S}_{i} \cdot \mathbf{S}_{j}$.

First, we replace the local constraints of no doubly occupied sites by statistical Gutzwiller weights (see below) and use a MF decoupling in the particle-hole channel to obtain a self-consistent renormalized MF Hamiltonian [7],

$$
\begin{aligned}
H_{\mathrm{MF}}= & -t \sum_{\langle i j\rangle \sigma} g_{i j}^{t}\left(c_{i, \sigma}^{\dagger} c_{j, \sigma}+\text { h.c. }\right) \\
& -\frac{3}{4} J \sum_{\langle i j\rangle \sigma} g_{i j}^{J}\left(\chi_{j i} c_{i, \sigma}^{\dagger} c_{j, \sigma}+\text { h.c. }-\left|\chi_{i j}\right|^{2}\right),
\end{aligned}
$$

where $\chi_{j i}=\left\langle c_{j, \sigma}^{\dagger} c_{i, \sigma}\right\rangle$ and where we explicitly assume an inhomogeneous solution and hence inhomogeneous Gutzwiller factors,

$$
\begin{aligned}
g_{i j}^{J} & =\frac{4\left(1-x_{i}\right)\left(1-x_{j}\right)}{\left(1-x_{i}^{2}\right)\left(1-x_{j}^{2}\right)-8 x_{i} x_{j}\left|\chi_{i j}\right|^{2}+16\left|\chi_{i j}\right|^{4}}, \\
g_{i j}^{t} & =\left[\frac{4 x_{i} x_{j}\left(1-x_{i}\right)\left(1-x_{j}\right)}{\left(1-x_{i}^{2}\right)\left(1-x_{j}^{2}\right)+8\left(1-x_{i} x_{j}\right)\left|\chi_{i j}\right|^{2}+16\left|\chi_{i j}\right|^{4}}\right]^{1 / 2},
\end{aligned}
$$


where $x_{i}, x_{j}$ are the local hole densities. The $\left|\chi_{i j}\right|$ terms account for the correlations of the probabilities between nearest-neighbor sites [8] and were not included in the original MF treatment [3] of the inhomogeneous state. Our MF scheme applies to the "normal" phase. In contrast, an additional decoupling in the particleparticle channel leads to an RVB superconducting state [6].

Interestingly, the parent insulating RVB state can be viewed as a staggered flux state (SFP) [9-11] MF solution of the above Hamiltonian. We show here that, away from half-filling, the SFP spontaneously acquires (small) bond modulations.

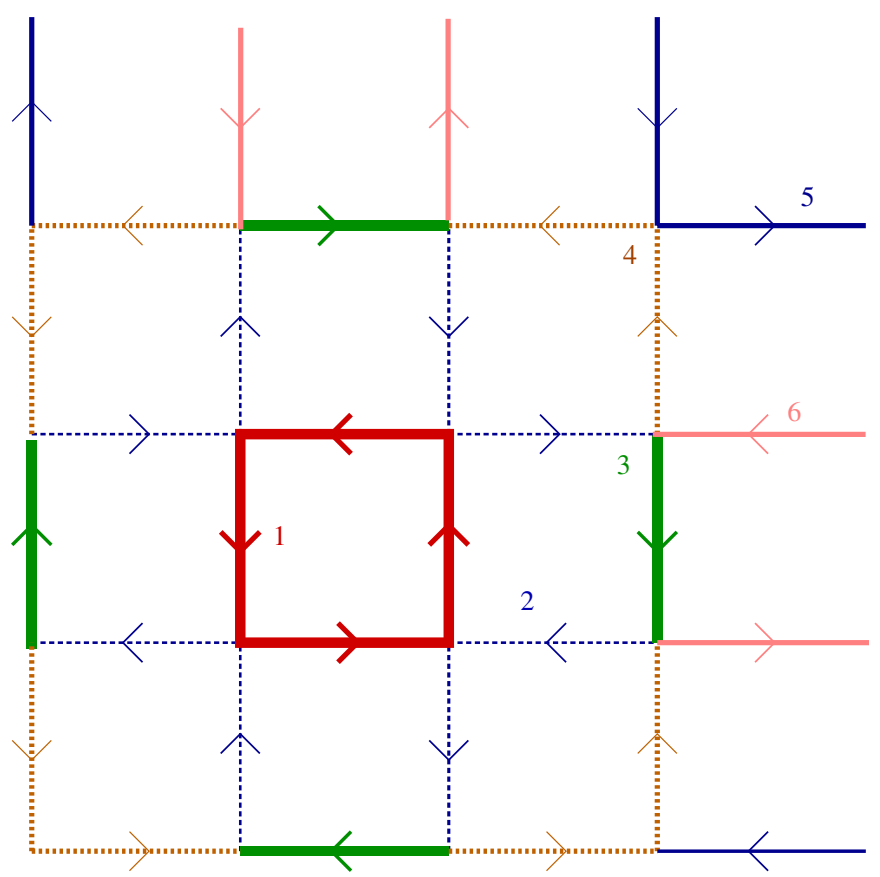

Fig. 1. Schematic pattern of the bond distribution. Non-equivalent bonds (labelled from 1 to 6 ) are indicated by different types of lines whose widths qualitatively reflect the bond magnitudes. Arrows indicate the directions of the small charge currents.

\section{Results and comparison with VMC}

Assuming a $4 \times 4$ supercell with overall rotational symmetry (see Fig. 1), the MF equations have been solved for an average doping of $\frac{1}{8}$. A typical solution depicted in Fig. 1 exhibits orbital currents according to the original SFP pattern. However, small modulations of the bond hoppings $\left\langle c_{i, \sigma}^{\dagger} c_{j, \sigma}\right\rangle$ and bond exchange terms $\left\langle\mathbf{S}_{i} \cdot \mathbf{S}_{j}\right\rangle$ as well as the on-site densities appear spontaneously. Numerical values of the bond (site) parameters for $t / J=3$ are given in Table 1 (Table 2). The charge-density-wave (CDW) modulation is found to be an order of magnitude smaller than the bond-order-wave (BOW) modulations. It was argued recently [4] that this instability is connected to nesting properties of the small ellipsoidal Fermi pockets of the SFP.

The MF results have been tested by an exact (within statistical errors) VMC energy minimization and evaluation of the order parameters (on a $16 \times 16$ lattice with mixed boundary conditions, see Ref. [5] for details). Comparative values are shown in Tables 1 and 2 . Interestingly, the VMC simulation also predicts a dominant BOW component w.r.t the CDW one. However, an overall reduction of the magnitudes of these modulations by an approximate factor of 4 compared to MF is seen.

How such a state can compete with the d-wave RVB superconductor is still unclear. However, our calculations $[3,5]$ have suggested that the BOW SFP is less sensitive

Table 2

Numerical values of the charge densities on the three non-equivalent sites (labelled from the center of the $4 \times 4$ pattern) for $t / J=3$ and doping $x \simeq \frac{1}{8}$

\begin{tabular}{llllll}
\hline Site \# & 1 & 2 & 3 & $n$ & $\%( \pm)$ \\
\hline MF (1) & 0.925 & 0.864 & 0.857 & 0.8776 & 4 \\
MF (2) & 0.901 & 0.898 & 0.806 & 0.8759 & 5.5 \\
VMC & $0.886(3)$ & $0.874(5)$ & $0.863(6)$ & 0.875 & 1.3 \\
\hline
\end{tabular}

Average density and relative amplitudes (in \%) are provided in the last column.

Table 1

Numerical values of the spin-spin correlations (first three lines) and of the real (next three lines) and imaginary (last three lines) parts of the bond hoppings $\left\langle\sum_{\sigma} c_{i \sigma}^{\dagger} c_{j_{\sigma}}\right\rangle$ in the BOW staggered flux state for $t / J=3$ and $x \simeq \frac{1}{8}$ (see Table 2 for exact densities)

\begin{tabular}{llllllll}
\hline Bond \# & 1 & 2 & 3 & 4 & 5 & 6 & Aver. \\
\hline MF (1) & -0.346 & -0.172 & -0.313 & -0.236 & -0.164 & -0.125 & -0.220 \\
MF (2) & -0.258 & -0.221 & -0.310 & -0.194 & -0.170 & -0.184 & -0.219 \\
VMC & -0.2149 & -0.2062 & -0.2128 & -0.1871 & -0.1810 & -0.1750 & -0.1963 \\
MF (1) & 0.055 & 0.057 & 0.119 & 0.106 & 0.092 & 0.073 & 0.051 \\
MF (2) & 0.057 & 0.054 & 0.061 & 0.081 & 0.112 & 0.053 & 0.069 \\
VMC & 0.0706 & 0.0742 & 0.0840 & 0.0833 & 0.0855 & 0.0825 & 0.0797 \\
MF (1) & 0.045 & 0.037 & 0.037 & 0.031 & 0.023 & 0.030 & 0.034 \\
MF (2) & 0.038 & 0.038 & 0.044 & 0.031 & 0.011 & 0.036 & 0.033 \\
VMC & 0.0400 & 0.0392 & 0.0414 & 0.0349 & 0.0313 & 0.0330 & 0.0367 \\
\hline
\end{tabular}

(1) From Ref. [3]; (2) with corrections in Gutzwiller factors. Two last columns: average and relative BOW modulations (in \%). 
than the superconducting state to a moderate (e.g. nearestneighbor) Coulomb repulsion. In this competition, the role of the disorder and/or the surface might be crucial.

\section{References}

[1] M. Vershinin, S. Misra, S. Ono, Y. Abe, Y. Ando, A. Yazdani, Science 303 (2004) 1995.

[2] T. Hanaguri, et al., Nature 430 (2004) 1001.

[3] D. Poilblanc, Phys. Rev. B 72 (2005) 060508(R).

[4] C. Li, S. Zhou, Z. Wang, Phys. Rev. B 73 (2006) 060501(R); for related results based on an instability critirium see also, Z. Wang, G. Kotliar, X.F. Wang, Phys. Rev. B 42 (1990) 8690.
[5] C. Weber, D. Poilblanc, S. Capponi, F. Mila, C. Jaudet, cond-mat/ 0601301.

[6] P.W. Anderson, Science 235 (1987) 1196.

[7] F.C. Zhang, C. Gros, T.M. Rice, H. Shiba, Supercond. Sci. Technol. 1 (1988) 36.

[8] T.C. Hsu, Phys. Rev. B 41 (1990) 11379;

M. Sigrist, T.M. Rice, F.C. Zhang, Phys. Rev. B 49 (1994) 12058.

[9] I. Affleck, J.B. Marston, Phys. Rev. B 37 (1988) R3774; J.B. Marston, I. Affleck, Phys. Rev. B 39 (1989) 11538.

[10] G. Kotliar, Phys. Rev. B 37 (1988) 3664.

[11] D. Poilblanc, Y. Hasegawa, Phys. Rev. B 41 (1990) 6989; M.U. Ubbens, P.A. Lee, Phys. Rev. B 46 (1992) 8434; P.A. Lee, N. Nagaosa, T. Ng, X. Wen, Phys. Rev. B 57 (1998) 6003; D.A. Ivanov, Phys. Rev. B 70 (2004) 104503. 